\title{
On the harmonic solution for plate bending: considering the effects of shear deformation and forces in the middle plane
}

\author{
L. Palermo Jr. \\ School of Civil Engineering, Architecture and Urban Design, \\ State University of Campinas, Brazil
}

\begin{abstract}
The solution for plate bending under harmonic loads including the geometrical non-linearity effect is presented. The influence of shear deformation and the rotatory inertia in the plate behavior were considered by using the elastodynamic fundamental solution. An additional domain integral was included in the boundary integral equations to consider the geometrical non-linearity effect where in-plane forces were assumed invariant with time and the deflection derivatives dependent of the harmonic solution. An analysis to get the lowest eigenvalue is used to show the relation between maximum values for in-plane forces according to the frequency value. Results obtained when plate rotations were used instead of deflection derivatives in the term related to the geometrical non-linearity effect are discussed and compared to solutions in the literature to show the extension of this strategy.

Keywords: harmonic solution, critical in-plane loads, the geometrical nonlinearity effect in Mindlin plates.
\end{abstract}

\section{Introduction}

The plate bending model including the effect of shear deformation is very efficient with reference to the classical bending model when it is necessary to evaluate stress concentration in the edge zone of the plate or around holes that have a diameter not larger than eight times the plate thickness [1]. The discrepancies in dynamic analysis using the classical model with reference to three-dimensional theory appeared for wavelengths less than 10 times the plate thickness and a limit for velocities could not be established for wavelengths approaching zero [2]. 
Mindlin presented a plate bending model similar to that proposed by Reissner [3] and included the influence of rotatory inertia to perform dynamic analyses [2].

A review on the literature until 1989 on the buckling of thin rectangular plates under in-plane loads was done by Jones in [4]. Lei et al. [5] formulated an integral equation for geometrically nonlinear behavior of Reissner plates, where the domain integral was discretized into constant triangular cells. A similar study was developed by Purbolaksono and Aliabadi [6] but using constant rectangular cells. Baiz and Aliabadi [7] made an improvement in evaluating the effect of geometric nonlinearity on the plate bending analysis by implementing a 9-node quadrilateral cell and introducing a free term factor in the integral equation. Karam and Telles [8] and Ribeiro and Venturini [9] used the domain discretization in dealing effects due to material nonlinearities.

Plate bending analyses for harmonic loads can be performed using the elastodynamic fundamental solution [10-12] in the boundary integral equations (BIEs). The effects of shear deformation and rotatory inertia can be considered alone or coupled in the elastodynamic fundamental solution [13] adopted in this study where an analysis on the solution for plate bending under harmonic loads considering the geometrical non-linearity effect is carried out. An additional domain integral was included in BIEs to consider the geometrical non-linearity effect with in-plane forces assumed invariant with time and the deflection derivatives dependent on the harmonic solution. An analysis to get the lowest eigenvalue is used to show the relation between maximum values for in-plane forces according to the frequency value. The numerical implementation used isoparametric quadratic boundary elements combined with constant cells on domain whereas the inverse iteration and Rayleigh quotient were employed in the eigenvalue analysis [14]. Results obtained when plate rotations were used instead of deflection derivatives in the term related to the geometrical non-linearity effect in BIEs are discussed and compared with solutions in the literature to show the extension of this strategy.

\section{Boundary integral equations}

The equations of motion in time-harmonic problems for an infinitesimal plate element under a transverse distributed loading $\mathrm{q}\left(\mathrm{t}, \mathrm{x}_{\mathrm{i}}\right)$ and considering in-plane forces [1] are next written with Latin indices taking values $\{1,2$ and 3$\}$ and Greek indices taking values $\{1,2\}$ :

$$
\begin{gathered}
M_{\alpha \beta, \beta}-Q_{\alpha}=\frac{\rho h^{3}}{12} \frac{\partial^{2} \psi_{\alpha}}{\partial t^{2}} \\
Q_{\alpha, \alpha}+q+\frac{\partial}{\partial x_{\alpha}}\left(N_{\alpha \beta} \frac{\partial w}{\partial x_{\beta}}\right)=\rho h \frac{\partial^{2} w}{\partial t^{2}}
\end{gathered}
$$

The in-plane forces $\mathrm{N}_{\alpha \beta}$, related to the plane stress problem, are assumed to be invariant with time whereas only the deflection derivatives are dependent of the harmonic solution, the plate has a uniform thickness $h$ and the mass density $\rho$. The transverse acceleration $\partial^{2} \mathrm{w} / \partial \mathrm{t}^{2}$ and the angular acceleration $\partial^{2} \psi_{\alpha} / \partial \mathrm{t}^{2}$ are functions of the deflection $\mathrm{w}$ and plate rotations $\psi_{\alpha}$, respectively. The constitutive relations are next written: 


$$
\begin{gathered}
M_{\alpha \beta}=D \frac{(1-v)}{2}\left(\psi_{\alpha, \beta}+\psi_{\beta, \alpha}+\frac{2 v}{1-v} \psi_{\gamma, \gamma} \delta_{\mathrm{a} \beta}\right) \\
Q_{\alpha}=D \frac{(1-v)}{2} \lambda^{2}\left(\psi_{\alpha}+w_{, \alpha}\right)
\end{gathered}
$$

with

$$
\lambda^{2}=12 \frac{\kappa^{2}}{h^{2}}
$$

$\mathrm{D}$ is the flexural rigidity, $v$ is the Poisson ratio. The shear parameter $\kappa^{2}$ is equal to $\pi^{2} / 12$ in this study $[2,12]$.

The static-like form of the equations of motion for harmonic problems led to a displacement boundary integral equation (DBIE) similar to that presented for static problems [10] but used in conjunction with the elastodynamic fundamental solution instead. The geometrical non-linearity effect is included thorough an additional domain integral in the DBIE:

$$
\begin{gathered}
\frac{1}{2} C_{i j}\left(x^{\prime}\right) u_{j}\left(x^{\prime}\right)+\int_{\Gamma}\left[T_{i j}\left(x^{\prime}, x\right) u_{j}(x)-U_{i j}\left(x^{\prime}, x\right) t_{j}(x)\right] d \Gamma(x) \\
=\iint_{\Omega} U_{i 3}\left(x^{\prime}, X\right)\left[q+\frac{\partial}{\partial X_{\alpha}}\left(N_{\alpha \beta} \frac{\partial u_{3}}{\partial X_{\beta}}\right)\right] d \Omega(X)
\end{gathered}
$$

$\mathrm{C}_{\mathrm{ij}}$ is an element of the matrix $\mathrm{C}$ related to the boundary at the source point, which becomes the identity matrix when a smooth boundary is considered. $\mathrm{u}_{\alpha}$ is $\psi_{\alpha}, \mathrm{u}_{3}$ is $\mathrm{w}, \mathrm{t}_{\alpha}$ is the traction force related to moments $\left(\mathrm{t}_{\alpha}=\mathrm{M}_{\alpha \beta} \cdot \mathrm{n}_{\beta}\right), \mathrm{t}_{3}$ is the force related to shear $\left(t_{3}=Q_{\alpha} \cdot n_{\alpha}\right)$. $U_{i j}$ represents the rotation $(j=1,2)$ or the deflection $(j=3)$ due to a unit couple $(\mathrm{i}=1,2)$ or a unit point force $(\mathrm{i}=3)$, respectively, $\mathrm{T}_{\mathrm{ij}}$ represents the moment $(j=1,2)$ or the shear $(j=3)$ due to a unit couple $(i=1,2)$ or a unit point force $(\mathrm{i}=3)$, respectively.

The elastodynamic fundamental solution in this study [13] was an improvement of [12] because the behavior of singularities in the fundamental solution kernels of DBIEs were turned to the same type of those existing in the elastostatic fundamental solution. Furthermore, the fundamental solution can be reduced to that for the classical plate theory [15] when variables related to the effects of shear deformation (S) and rotatory inertia (R) are set equal to zero, as in [12]. The variables in DBIE, equation (5), related to the fundamental solution are next written in terms of the potential functions $\phi_{\mathrm{i} \beta}$ and $\mathrm{H}_{\mathrm{i}}$ :

$$
\begin{gathered}
U_{i 3}=-\left(\beta_{1}+1\right) \phi_{i 1}\left(\delta_{1} r\right)-\left(\beta_{1}+1\right) \phi_{i 2}\left(\delta_{2} r\right) \\
U_{i \alpha}=\frac{\partial}{\partial x_{\alpha}}\left[\phi_{i 1}\left(\delta_{1} r\right)+\phi_{i 2}\left(\delta_{2} r\right)\right]+e_{3 \alpha \beta} \frac{\partial}{\partial x_{\beta}}\left[H_{i}\left(\delta_{3} r\right)\right]
\end{gathered}
$$

$e_{3 \alpha \beta}$ is the permutation symbol. The arguments of the potential functions are dependent of variables related to the rotatory inertia $(\mathrm{R})$, the shear deformation $(\mathrm{S})$ and the classical dynamic factor for plates $\left(\delta_{0}{ }^{4}\right)$, defined in [2]:

$$
R=\frac{h^{2}}{12} ; \quad S=\frac{1}{\lambda^{2}} \frac{2}{(1-v)} ; \quad \delta_{0}^{4}=\frac{\omega^{2} \rho h}{D}
$$

The potential functions of the solution due to the unit point force are:

$$
\phi_{31}=-\frac{1}{2 \pi D} \frac{1}{\left(\delta_{1}^{2}-\delta_{2}^{2}\right)} K_{0}\left(i \delta_{1} r\right) ; \quad \phi_{32}=\frac{1}{2 \pi D} \frac{1}{\left(\delta_{1}^{2}-\delta_{2}^{2}\right)} K_{0}\left(i \delta_{2} r\right) ; \quad H_{3}=0 .
$$


When the solution due to unit couple in direction $\gamma$ is used, they are given by:

$$
\begin{gathered}
\phi_{\gamma 1}=-\frac{1}{2 \pi D} \frac{1}{\left(\delta_{1}^{2}-\delta_{2}^{2}\right)} \frac{\partial}{\partial x_{\gamma}}\left[K_{0}\left(i \delta_{1} r\right)+\ln (r)\right] \\
\phi_{\gamma 2}=\frac{1}{2 \pi D} \frac{1}{\left(\delta_{1}^{2}-\delta_{2}^{2}\right)} \frac{\partial}{\partial x_{\gamma}}\left[K_{0}\left(i \delta_{2} r\right)+\ln (r)\right] \\
H_{\gamma}=-e_{3 \gamma \eta} \frac{1}{2 \pi D} \frac{1}{\delta_{3}^{2}} \frac{2}{(1-v)} \frac{\partial}{\partial x_{\eta}}\left[K_{0}\left(i \delta_{3} r\right)+\ln (r)\right] .
\end{gathered}
$$

with

$$
\begin{gathered}
\delta_{1}^{2}=\frac{1}{2} \delta_{0}^{4}\left(R+S+\sqrt{(R-S)^{2}+\frac{4}{\delta_{0}^{4}}}\right) ; \quad \delta_{2}^{2}=\frac{1}{2} \delta_{0}^{4}\left(R+S-\sqrt{(R-S)^{2}+\frac{4}{\delta_{0}^{4}}}\right) \\
\delta_{3}^{2}=\lambda^{2}\left(R S \delta_{0}^{4}-1\right) ; \quad \beta_{1}=S\left(\delta_{1}^{2}-\delta_{0}^{4} R\right) ; \quad \beta_{2}=S\left(\delta_{2}^{2}-\delta_{0}^{4} R\right) .
\end{gathered}
$$

The equations of motion for in-plane forces invariant with time are equal to the equilibrium equations in static problems $\left(\mathrm{N}_{\alpha \beta, \alpha}=0\right)$ and some authors label this type of in-plane forces as "static in-plane forces" in dynamic analyses. The term related to the geometrical non-linearity effect in equations (2) or (5) can be simplified when the equilibrium equations for in-plane forces in static problems are used. The curvatures or the second derivatives for deflection appear as result from this simplification, as shown in several papers in the literature. This simplification was not used in this study but an algebraic manipulation was done in the domain integral related to in-plane forces of equation (5) to employ the divergence theorem.

$$
\begin{gathered}
\iint_{\Omega} U_{i 3}\left(x^{\prime}, X\right)\left[\frac{\partial}{\partial X_{\alpha}}\left(N_{\alpha \beta} \frac{\partial u_{3}(X)}{\partial X_{\beta}}\right)\right] d \Omega(X)= \\
\int_{\Gamma} n_{\alpha}(x) N_{\alpha \beta}(x) u_{3, \beta}(x) U_{i 3}\left(x^{\prime}, x\right) d \Gamma(x)-\iint_{\Omega} N_{\alpha \beta}(X) u_{3, \beta}(X) U_{i 3, \alpha}\left(x^{\prime}, X\right) d \Omega(X)
\end{gathered}
$$

The algebraic manipulation carried to using deflection gradients, only, when the geometrical non-linearity effect is considered. The final DBIE is given by:

$$
\begin{gathered}
\quad \frac{1}{2} C_{i j}\left(x^{\prime}\right) u_{j}\left(x^{\prime}\right)+\int_{\Gamma}\left[T_{i j}\left(x^{\prime}, x\right) u_{j}(x)-U_{i j}\left(x^{\prime}, x\right) t_{j}(x)\right] d \Gamma(x)= \\
=\iint_{\Omega} q(X) U_{i 3}\left(x^{\prime}, X\right) d \Omega(X)+\int_{\Gamma} n_{\alpha}(x) N_{\alpha \beta}(x) u_{3, \beta}(x) U_{i 3}\left(x^{\prime}, x\right) d \Gamma(x)+ \\
-\iint_{\Omega} N_{\alpha \beta}(X) u_{3, \beta}(X) U_{i 3, \alpha}\left(x^{\prime}, X\right) d \Omega(X)
\end{gathered}
$$

The gradient BIE at an internal point is obtained by differentiating the displacement BIE with respect to the coordinate of the source point ( $\left.\mathrm{X}^{\prime}\right)$. The result for the deflection gradient BIE can be written using differentiation in terms of the coordinate of the field point with the direction cosines of the outward normal at the field point written off the differential operator in the notation [17]:

$$
\begin{gathered}
u_{3, \gamma}\left(X^{\prime}\right)=\int_{\Gamma}\left\{n_{\alpha}(x) \frac{\partial}{\partial x_{\gamma}}\left[M_{3 \alpha \beta}\left(X^{\prime}, x\right)\right] u_{\beta}(x)+\cdots\right. \\
\ldots+n_{\beta}(x) \frac{\partial}{\partial x_{\gamma}}\left[Q_{3 \beta}\left(X^{\prime}, x\right)\right] u_{3}(x)-\frac{\partial}{\partial x_{\gamma}}\left[U_{3 \beta}\left(X^{\prime}, x\right)\right] t_{\beta}(x)+\cdots \\
\left.\ldots-\frac{\partial}{\partial x_{\gamma}}\left[U_{33}\left(X^{\prime}, x\right)\right] t_{3}(x)\right\} d \Gamma(x)-\iint_{\Omega} \frac{\partial}{\partial x_{\gamma}}\left[U_{33}\left(X^{\prime}, X\right)\right] q(X) d \Omega(X) \ldots
\end{gathered}
$$




$$
\begin{gathered}
\ldots-\int_{\Gamma} n_{\alpha}(x) N_{\alpha \beta}(x) u_{3, \beta}(x) \frac{\partial}{\partial x_{\gamma}}\left[U_{i 3}\left(X^{\prime}, x\right)\right] d \Gamma(x)+\ldots \\
\ldots+\iint_{\Omega} N_{\alpha \beta}(X) u_{3, \beta}(X) \frac{\partial}{\partial X_{\gamma}}\left[U_{i 3, \alpha}\left(X^{\prime}, X\right)\right] d \Omega(X)
\end{gathered}
$$

\section{Numerical implementation}

The numerical implementation employed quadratic shape functions for isoparametric boundary elements with collocation points always placed on the boundary. The same mapping function was used for conformal and non-conformal interpolations, i.e. nodes at ends of quadratic elements remain at ends when discontinuous elements were employed. The collocation points were placed at nodes in case of continuous elements and at positions $(-0.67,0.0,+0.67)$, in the range $(-1,1)$, in case of discontinuous elements, i.e. the collocation points were shifted to inside the element at the corresponding end where the discontinuity exists. Constant rectangular cell elements were used to discretize the domain integral related to the geometrical non-linearity effect, which was converted into an equivalent boundary integral performed on each side of the cell. The singularity subtraction [16] and the transformation of variable technique [18] were employed for the Cauchy and the weak type singularity, respectively, when integrations were performed on elements (or, side of the cell) containing the collocation points. The standard Gauss-Legendre scheme was employed for integrations on elements (or, side of the cell) not containing the collocation points. The inverse iteration and the Rayleigh quotient were used to perform the eigenvalue analysis. The basic inverse iteration procedure was employed without using the shift of origin proposed by Wielandt [14], i.e.:

$$
\begin{gathered}
A x^{(k+1)}=x^{k} \\
\lambda_{k}=\frac{\left(x^{(k+1)}, x^{k}\right)}{\left(x^{(k+1)}, x^{(k+1)}\right)}
\end{gathered}
$$

The basic inverse iteration procedure is very efficient for symmetric matrices and there are a lot of recommendations in [14] for complex matrices as well as on the procedure to introduce the shift of the origin to improve the convergence rate. In this study, the use of the basic procedure to obtain the lowest eigenvalue had a low number of iteration, which was in the range 6 to 14. The dot product between complex eigenvectors $\mathrm{x}^{\mathrm{k}+1}$ and $\mathrm{x}^{\mathrm{k}}$ at the iteration $\mathrm{k}$ was done in the Rayleigh quotient according to equation (11). Equation (10) was not used explicitly in this study but starting with an eigenvector $\mathrm{x}^{1}$ with all elements (deflection gradients in directions $\mathrm{x}_{1}$ and $\mathrm{x}_{2}$ at the center of each cell) equal to 1.0, values for displacements and tractions at nodes of the boundary elements were found. These values were introduced in the discretized form of equation (9), i.e. the equation written in terms of matrices, to obtain the eigenvector $\mathrm{x}^{2}$ and the lowest eigenvalue at the first iteration step was obtained by using equation (11). The iteration procedure continued until the absolute difference between values of successive eigenvalues was less than $10^{-5}$. The proof of the convergence for the lowest eigenvalue can be found in [14]. The use of the Rayleigh quotient to obtain eigenvalues from low 
precision eigenvectors was shown in [19] for symmetric positive definite matrices where the simple precision ( 4 bytes in a FORTRAN code) was adopted in the code to define the eigenvectors precision. The basic inverse iteration was used in [20, 21] to obtain the critical in-plane forces and the natural frequencies for plates according to the classical bending model in the boundary element method with the static fundamental solution.

The considered plate in this study was the same as that adopted in $[12,13]$ to obtain the natural frequencies when the ratio $(\mathrm{h} / \mathrm{a})$ between the thickness value $(\mathrm{h})$ and the plate side value (a) was increased from 0.05 to 1.0. The Young modulus (E) was $206.9 \mathrm{GPa}$, the Poisson ration ( $v$ ) was 0.3 , the mass density ( $\rho$ ) was $7860 \mathrm{~kg} \cdot \mathrm{m}^{-3}$, the side of the plate (a) had a length equal to $50 \mathrm{~cm}$. Three values for the ratios $(\mathrm{h} / \mathrm{a})$ were used, $0.01,0.05$ and 0.10 to analyze the geometrical nonlinearity effect because thick plates would be obtained for greater ratios. The use of complex values in the fundamental solution does not limit the value for thickness in this boundary element formulation as explained in [12, 13]. The natural frequencies obtained in $[12,13]$ are next written and the theoretical values according to the classical theory were included:

Table 1: $\quad$ First natural frequencies $(\mathrm{rad} / \mathrm{s})$.

\begin{tabular}{|c|c|c|c|}
\hline Ratio h/a & Classical [12] & Mindlin [22] & {$[13]$} \\
\hline 0.01 & 612.8 & 610 & - \\
\hline 0.05 & 3064 & 3049 & 3015 \\
\hline 0.10 & 6128 & 5918 & 5800 \\
\hline
\end{tabular}

The natural frequencies in Table 1 were obtained for the plate simply supported from all sides with hard condition (twisting moments restrained along the side). The first analysis was the computation of maximum values for in-plane forces applied in one direction, i.e. on edges of opposite sides of the plate. The adopted frequency in the elastodynamic solution was $0.1 \mathrm{rad} / \mathrm{s}$ to approach the static condition and two meshes were used with two nodes at each corner:

a) 32 quadratic boundary elements (68 nodes) and 16 constant cells;

b) 64 quadratic boundary elements (132 nodes) and 64 constant cells.

Table 2: $\quad$ First critical in-plane force $(\mathrm{KN} / \mathrm{m})$ - with gradient of deflections.

\begin{tabular}{|c|r|r|r|r|r|}
\hline Ratio h/a & Classical & $\begin{array}{c}\text { Shear and } \\
\text { Rotatory }\end{array}$ & $\begin{array}{c}32 \mathrm{BE} \\
16 \text { cell } \\
\text { Full }\end{array}$ & $\begin{array}{c}64 \mathrm{BE} \\
64 \text { cell } \\
\text { Full }\end{array}$ & $\begin{array}{c}32 \mathrm{BE} \\
16 \text { cell } \\
\text { Sym. }\end{array}$ \\
\hline 0.01 & 376.5 & 376.3 & 391.3 & 378.5 & 379.2 \\
\hline 0.05 & 47064.5 & 46400.8 & 48174.6 & 46662.6 & 46668.0 \\
\hline 0.10 & 376515.7 & 356146.2 & 369386.5 & 357989.4 & 358003.3 \\
\hline
\end{tabular}


The values in the row "Classical" in Table 2 were computed using the solution in [1] and those in the row "Shear and Rotatory" were obtained using the vibration modes and procedures presented by Mindlin to obtain natural frequencies in [22]. The theoretical values were obtained for compression forces because the frequency is near to zero. The difference in theoretical values for critical in-plane forces was not significant for the ratio equals to 0.01 when the effect of shear deformation was considered. Values obtained with the second mesh (64 BE and 64 cells) were close to theoretical values as well as for those obtained when a quarter of the plate was discretized using the first mesh ( $32 \mathrm{BE}$ and 16 cells) and employing the symmetry condition as shown in results of the last row. The reduction in the difference when the thickness was increased (ratios 0.05 and 0.10 ) can be attributed to the introduction of the effect of shear deformation in the computation for all values and a numerical instability is being introduced for the lowest ratio.

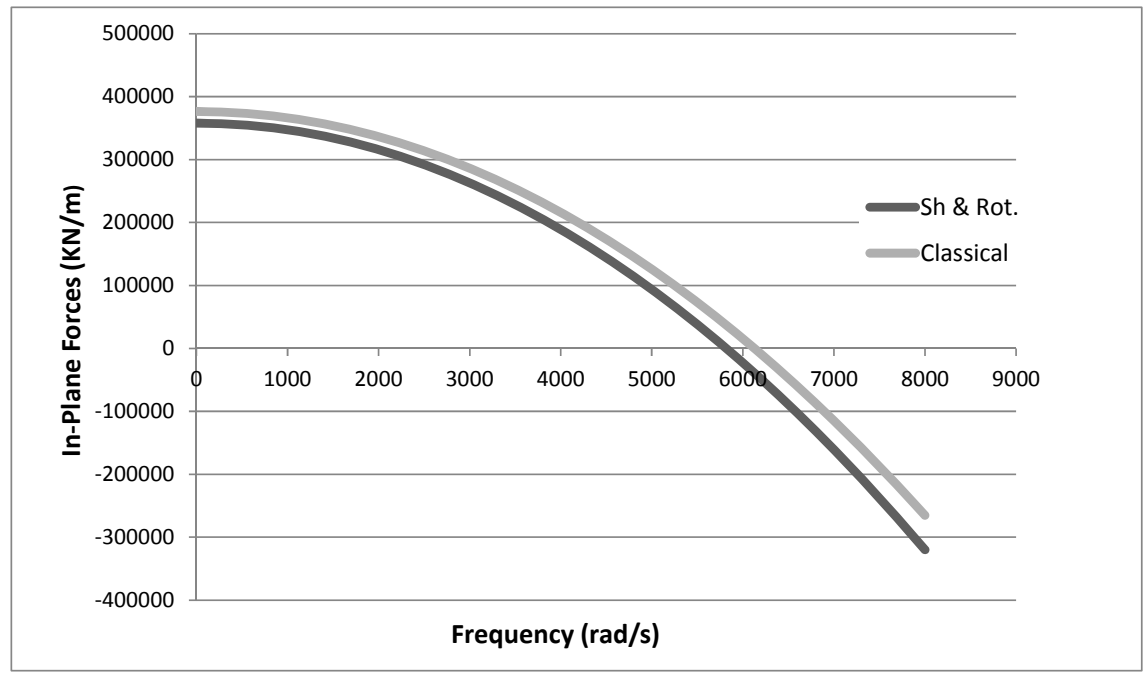

Figure 1: Maximum in-plane forces according to the frequency for a square plate simply supported from all sides with $\mathrm{h} / \mathrm{a}=0.1$.

The changing in the maximum value for in-plane forces according to the frequency is shown in Figure 1 where the lowest eigenvalue was obtained when the frequency value was increased. The inverse iteration seeks the lowest absolute value for eigenvalue and the code adopted positive values for compression inplane forces. It can be noted that tension values for in-plane forces were obtained for frequency values greater than first natural frequency. The effect of shear deformation introduced a reduction in value of the in-plane force that was increased according to the frequency. The behavior shown in Figure 1 can be changed according to the boundary value problem. A non-significant reduction in value of the in-plane force when the shear deformation is considered is shown in Figure 2. The plate simply remained, supported on opposite sides with in-plane 
forces applied on these edges whereas the edges without in-plane forces were changed to free and clamped, respectively. The analysis of the frequency response $[12,13]$ to find the first natural frequency for the ratio (h/a) equals to 0.1 carried to a value of $3800 \mathrm{rad} / \mathrm{s}$ when the effects of shear deformation and rotatory inertia were considered and $3850 \mathrm{rad} / \mathrm{s}$ using the classical model. The second mesh was used (64 BE and 64 cells). This type of boundary condition appears in flange elements of beam sections of steel structures.

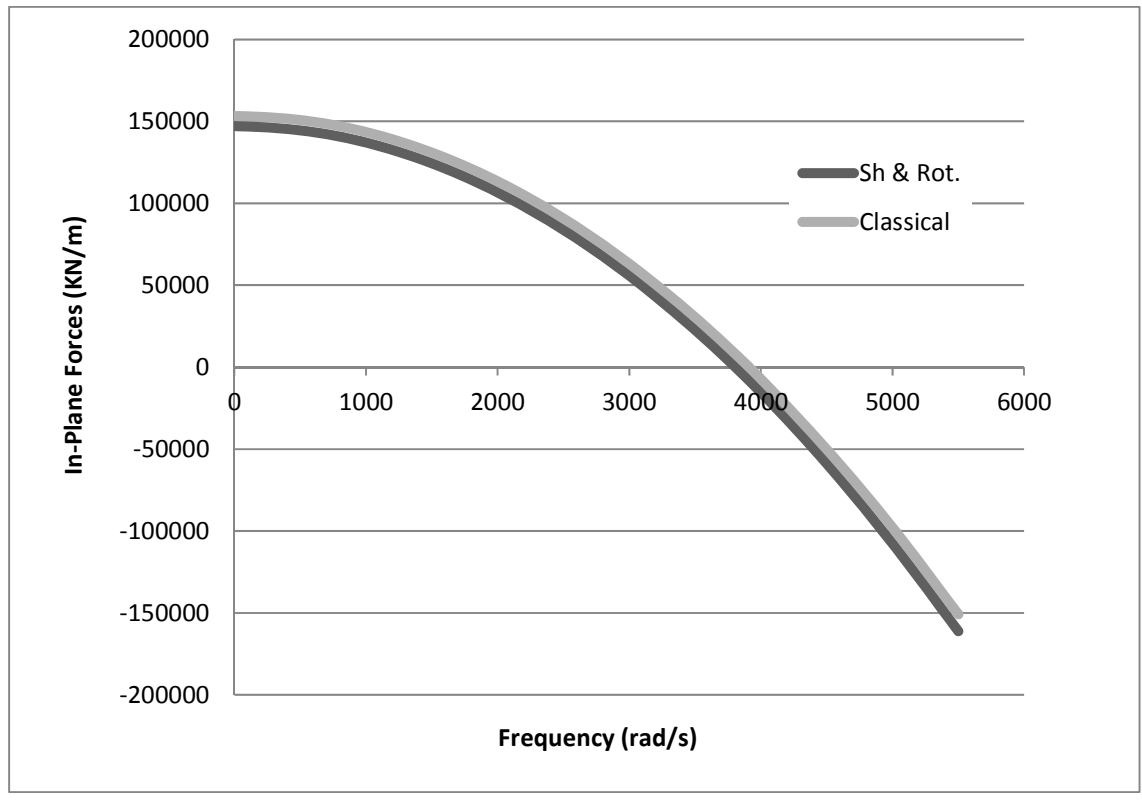

Figure 2: Maximum in-plane forces according to the frequency for a square plate simply supported on opposite sides, one edge free and other clamped with $\mathrm{h} / \mathrm{a}=0.1$.

Table 3: $\quad$ First critical in-plane force $(\mathrm{KN} / \mathrm{m})-$ with plate rotations.

\begin{tabular}{|c|r|r|r|r|r|}
\hline \multirow{2}{*}{ Ratio h/a } & \multirow{2}{*}{$\begin{array}{c}\text { Shear and } \\
\text { rotatory }\end{array}$} & \begin{tabular}{c} 
6radients \\
of \\
\cline { 3 - 6 }
\end{tabular} & $\begin{array}{c}\text { Geflection } \\
\text { Plate }\end{array}$ & $\begin{array}{c}\text { Gradients } \\
\text { of } \\
\text { rotations }\end{array}$ & $\begin{array}{c}\text { Plate } \\
\text { rotations }\end{array}$ \\
\hline 0.01 & 376.3 & 378.5 & 378.7 & 379.2 & 379.4 \\
\hline 0.05 & 46400.8 & 46662.6 & 47338.5 & 46668.0 & 47343.3 \\
\hline 0.10 & 356146.2 & 357989.4 & 378708.4 & 358003.3 & 378719.8 \\
\hline
\end{tabular}

When the gradients of deflection were replaced in the domain integral of BIEs by plate rotations multiplied by -1 the results were closer for theoretical values when the ratio 0.01 was used (as shown in Table 3 ). The equation for gradients, equation (9), is not used in this strategy and rotations are obtained with the 
displacement BIEs. The theoretical value for the lowest ratio $(0.01)$ is the almost the same when the shear deformation is considered or not. Thus, the use of plate rotations can be interesting when values near to the classical theory are being required. Furthermore, this behavior is consistent with the introduction of the effect of shear deformation that decouples plate rotations from gradients of deflection when thickness is increased [22].

\section{Conclusions}

The results obtained in solutions for harmonic loads including the geometrical non-linearity effect and employing the elastodynamic fundamental solution [13] were consistent with the expected behavior for plates according to the literature. The features shown and discussed using this formulation can be employed for other plate analyses according to specific requirements. The elastodynamic fundamental solution [13] presented a stable behavior in the analyses.

\section{Acknowledgements}

The author is grateful to CNPq and FAPESP for support the development of the research on plates.

\section{References}

[1] Timoshenko, S. P., Woinowsky-Krieger, S., Theory of Plates and Shells, McGraw-Hill Book Company, New York, 2nd Ed., 1959.

[2] Mindlin, R.D., Influence of rotatory inertia and shear on flexural motions of isotropic elastic plates, Journal of Applied Mechanics, 1951.

[3] Reissner, E., The Effect of Transverse Shear Deformation on the Bending of Elastic Plates, Journal of Applied Mechanics, 1945.

[4] Jones N. Structural impact. Cambridge, MA: Cambridge University Press; 1989.

[5] Lei, X.Y., Huang, M.K., Wang, X.X., Geometrically nonlinear analysis of a Reissner's type plate by boundary element method, Comput. Struct. 37 (6), pp. 911-916, 1990.

[6] Purbolaksono, J., Aliabadi, M.H., Large deformation of shear deformable plate by boundary element method. J. Eng. Math. 51, pp. 211-230, 2005.

[7] Baiz, P.M., Aliabadi, M.H., Large deflection analysis of shear deformable shallow shells by the field boundary element method. In: 6th Int. Conf. on Boundary Element Tech, 2005.

[8] Karam, V.J., Telles, J.C.F., Nonlinear material analysis of Reissner's plates. In: Plate Bending Analysis with Boundary Element. Computational Mechanics Publications, Southampton, pp. 127-163, 1998.

[9] Ribeiro, G.O., Venturini, W.S., Elastoplastic analysis of Reissner's plate using the boundary element method. In: Plate Bending Analysis with Boundary Element. Computational Mechanics Publications, Southampton, pp. 101-125, 1998. 
[10] Antes, H., Static and Dynamic Analysis of Reissner-Mindlin Plates, Boundary Element Analysis of Plates and Shells, Editor: D.E. Beskos, Springer Series in Computational Mechanics Edited by S.N. Atluri, Springer-Verlag, 1991.

[11] Palermo Jr., L., On the fundamental solution to perform the dynamic analysis of Reissner-Mindlin's plates, Boundary Element Technology XV, Editors C.A. Brebbia, R.E. Dippery, 2003.

[12] Palermo Jr., L., On the harmonic response of plates with the shear deformation effect using the elastodynamic solution in the boundary element method, Engineering Analysis with Boundary Elements, 2007.

[13] Palermo Jr., L., An alternative dynamic fundamental solution for plate bending including the shear deformation effect. In: Boundary Elements and other Mesh Reduction Methods XXXV, 2013, WIT Press, 2013.

[14] Wilkinson, J.H., The Algebraic Eigenvalue Problem, Oxford University Press, William Clowes \& Sons, London, 1972.

[15] Vivoli J, Filippi P. Eigenfrequencies of thin plates and layer potentials. J Acoust Soc Am; 55: pp. 562-7, 1974.

[16] Aliabadi, M.H., The boundary element method, applications in solids and structures, vol. 2, John Wiley \& Sons Ltd., 2002.

[17] Palermo Jr., L., The tangential differential operator applied to a stress boundary integral equation for plate bending including the shear deformation effect, Eng Anal Bound Elem, 36, pp. 1213-1225, 2012.

[18] Telles, J.C.F, A self-adaptive coordinate transformation for efficient numerical evaluation of general boundary element integrals, Int. J. Num. Meth. Eng., 24, pp. 959-973, 1987.

[19] Palermo Jr, L., Rachid, M., A Iteração Vetorial no Cálculo de Auto-Valores e Auto-Vetores, VII Congresso Latino Americano sobre Métodos Computacionais para Engenharia, São Carlos, 1986 (in Portuguese).

[20] Simões, R., Palermo Jr. L., O Cálculo das Menores Cargas Críticas em Placas Finas Usando o Método dos Elementos de Contorno, XXIX Jornadas Sul-Americanas de Ingenieria Estructural, Punta del Este, 2000 (in Portuguese).

[21] Simões, R., Palermo Jr, L., O Cálculo das Menores Freqüências Naturais em Placas Usando o Método dos Elementos de Contorno, 23. XXI Iberian Latin-American Congress on Computational Methods in Engineering, Campinas, 2001 (in Portuguese).

[22] Mindlin, R.D., Schacknow A., Deresiewicz, H., Flexural vibrations of rectangular plates, Journal of Applied Mechanics, 23, pp. 430-436, 1956. 